\title{
Preoperative anxiety: detection and contributing factors
}

Neal H. Badner MD, Warren R. Nielson PhD, Shirley Munk Msc, Caroline Kwiatkowska, Adrian W. Gelb MB ChB
The purpose of this study was to determine whether there is a correlation between anxiety the night before surgery and that existing immediately preoperatively, whether anaesthetists can detect preoperative anxiety and to establish the presence of any factors that might assist in the determination of preoperative anxiety. Anxiety was measured objectively using the Spielberger State-Trait Anxiety Inventory (STAI), and the Multiple Affect Adjective Check List (MAACL). Anxiety was found to be higher in females and those not having had a previous anaesthetic, and to remain constant from the afternoon before surgery to the immediate preoperative period. Anaesthetists were found to be poor assessors of anxiety unless they specifically questioned their patients about this.

Le but de cette étude est de déterminer s'il existe une corrélation entre l'anxiété la veille de la chirurgie et celle existant immédiatement en période préopératoire, si les anesthésistes peuvent dérecter l'anxiété préopératoire et établir la présence de facteurs qui peuvent assister à la détermination de cette anxiété. L'anxiété a été mesurée objectivement par le test de "Spielberger State-Trait Anxiery Inventory (STAI)" et le "Multiple Affect Adjective Check List (MAACL) ". L'anxiété fut trouvée supérieure chez les femmes ainsi que chez ceur qui n' ont pas eu d'anesthésie au préalable el fut trouvée constante à partir de l'après midi avant la chirurgie à la période préopératoire immédiate. Les anesthésistes furent trouvés comme des pauvres évaluateurs de l'anxiété sauf quand celle-cifut exprimée par les patients lors du questionnaire.

\section{Key words}

ANAESTHESIA: evaluation, preoperative; PREMEDICATION; PSYCHOLOGICAL RESPONSES.

From the Departments of Anaesthesia and Psychology, University Hospital, London, Ontario.

Address correspondence to: Dr. A.W. Gelb, Department of Anaesthesia, University Hospital, PO Box 5339, Postal Stn A, London, Ontario, Canada N6A 5A5
It has been shown that the majority of patients admitted to hospital for elective surgery experience anxiety preoperatively. ${ }^{1-3}$ Anxiety and stress are unpleasant sensations and can also adversely influence the surgical procedure as well as affect the patient's recovery. ${ }^{4,5}$ It is for these reasons that anaesthetists premedicate patients that they determine to be anxious.

A recent study demonstrated that anxiety on the afternoon before surgery predicted anxiety preoperatively. ${ }^{6}$ This makes the effectiveness of anxiolytic premedication critically dependent on the anaesthetist's ability to detect anxiety during the preoperative visit. This ability has not, however, been adequately verified using objective criteria. The aim of our study was threefold: to assess the ability of anaesthetists to detect and quantify preoperative anxiety, to substantiate the correlation between anxiety on the afternoon prior to surgery with that found in the immediate preoperative period, and to try to identify the factors that would enhance the ability to detect and quantify preoperative anxiety.

\section{Methods}

The research protocol was approved by our Institutional Review Board, and all patients gave written informed consent. Subjects included all patients booked for elective surgery. Patients were excluded for the following: age less than 18, inability to read or speak English, psychosis, and central neurological impairment.

Anaesthetists interviewed 96 patients the day before scheduled surgery. Immediately after completing the patient visit, the anaesthetist was asked to assess both the patient's present level of anxiety and predict the level of anxiety at the time of surgery, using a visual analogue scale. An excellent correlation between visual analogue scales and other objective tests of anxiety have previously been shown. ${ }^{7}$ The anaesthetist was also asked to indicate the symptoms and signs on which the assessment was based. At the same time, the patient's level of anxiety was quantified using both the Spielberger State-Trait Anxiety Inventory (STAI) and the Multiple Affect Adjective Check List (MAACL). The patient was also instructed to complete a second STAI-state anxiety questionnaire one hour preoperatively or prior to receiving any premedication whichever was earlier. 
An additional 200 patients, both in- and outpatients, were only asked to complete the STAI-state and the MAACL-state questionnaires in the immediate preoperative period but were not assessed by the anaesthetist. These were completed either one hour preoperatively or immediately before receiving a premedicant, whichever occurred first.

Both questionnaires measure trait anxiety (a personality characteristic) and state anxiety (anxiety level at a given moment). The Spielberger inventory consists of two 20item questionnaires, one for trait and one for state anxiety. Each of the 20 items is a statement and the subject must indicate the statement's applicability as being either not at all, somewhat, moderately so, or very much so. The questionnaires are scored so that a higher score (maximum 80) indicates increased anxiety. The MAACL is a list of 132 words with II "anxiety present" words scored one each if selected and ten "anxiety absent" words scored one each if not selected. The range of scores is therefore 0-21 with increasing score equating with increasing anxiety. The MAACL includes a similar scoring procedure for the assessment of depression and hostility.

Information regarding the patient's age, sex, surgical procedure, previous surgery, coexisting medical disease, and current medications were obtained from the hospital chart. This information was used to determine if various subpopulations existed. Patient age was classified as less than or greater than $40 \mathrm{yr}$ and surgical procedure according to surgical subspeciality, e.g., orthopaedics, gynaecology. The anaesthetists' scores were also subdivided based on the criteria used by each anaesthetist; whether it was physical characteristics (sweaty palms, tachycardia), visual attributes ("looks anxious/worried"), or direct questioning of the patient (patient asked if they were anxious or wanted some medication to relieve anxiety).

\section{Data analysis}

The anaesthetists' assessments were correlated with the results of the questionnaire using linear regression techniques. Statistically significant differences between the correlation coefficients were determined using Fisher's $\mathrm{r}-\mathrm{Z}$ transformation and independent $\mathrm{t}$ tests. To determine if age, sex, surgical procedure, or a history of previous surgery confounded this data, an analysis of variance was undertaken.

\section{Results}

Two hundred and eighty-four patients completed the Spielberger STAI questionnaire one hour before surgery and 200 the MAACL. Twelve of 96 patients assessed by anaesthetists did not complete the questionnaire in the immediate preoperative period due to $O R$ schedule

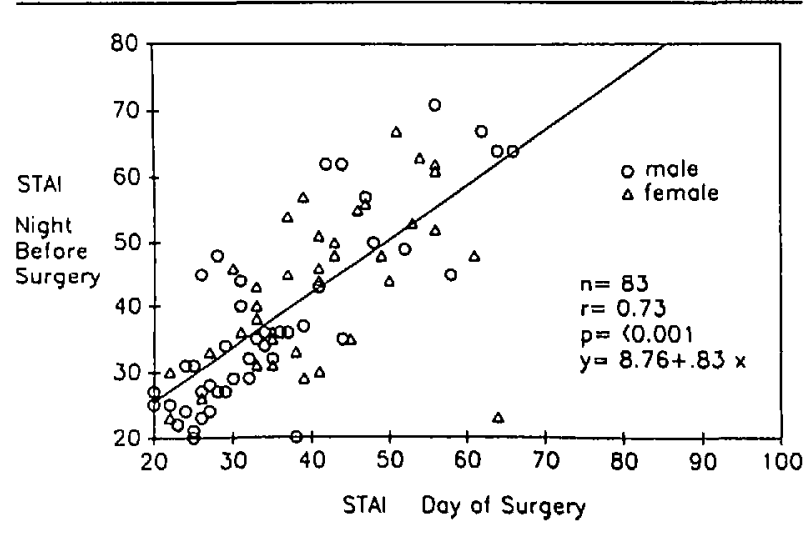

FIGURE Correlation between the STAI scores the aftemoon before surgery and those immediately preoperative.

changes. Table I summarizes the mean level of anxiety as a function of sex and previous anaesthesia. Anxiety was higher in females and those not having had a previous anaesthetic. Even those who rated their previous anaesthetic as unpleasant had a lower anxiety score than those who had never had an anaesthetic (41.0 \pm 13.1 vs $51.7 \pm$ $10.4, P<0.01)$. No difference was found between inpatients and outpatients or according to patient age.

Twenty-two anaesthetists (12 staff and ten residents) assessed 96 patients the afternoon preoperatively but complete data was only obtained in 84 patients (see above). Each anaesthetist assessed between two and six different patients. The patients included 39 females and 45 males with ages ranging from 18 to 73 . There was a significant correlation between the STAI and MAACL anxiety scores the afternoon before surgery $(r=0.53, P$ $<0.001)$. The Figure shows the correlation between the STAl scores the afternoon before surgery and those immediately preoperatively. A combined correlation co-

TABLE I Mean anxiety scores immediately preoperatively

\begin{tabular}{lll}
\hline \multicolumn{3}{c}{ Preoperative factor } \\
\cline { 2 - 2 } & \multicolumn{1}{c}{ STAI } & MAACL \\
\hline Sex & & \\
$\quad$ Male & $38.2 \pm 12.3$ & $64.7 \pm 27.5$ \\
Female & $42.9 \pm 12.8^{*}$ & $72.8 \pm 25.5^{*}$ \\
& & \\
Previous anaesthesia & & \\
Yes & $40.0 \pm 12.5$ & $67.8 \pm 25.5$ \\
No & $49.4 \pm 11.6 \dagger$ & $88.0 \pm 28.2 \dagger$ \\
\hline
\end{tabular}

Mean \pm SD. STAl $=$ Spielberger State Trait Anxiety Inventory state scores $(n=284)$.

MAACL $=$ Multiple Affect Adjective Check List state scores $(n=200)$. $* P<0.05$ males vs females.

$\dagger P<0.01$ previous vs no previous anaesthesia. 
TABLE II Correlations between anaesthetist assessment and afternoon test scores

Correlation coefficients

\begin{tabular}{llll}
\hline & \multicolumn{2}{l}{ Anaesthetists' scores } \\
\cline { 2 - 4 } State scores & Combined & Staff & Residents \\
\hline STAI & 0.28 & 0.33 & 0.23 \\
MAACL & 0.16 & 0.20 & 0.12 \\
\hline
\end{tabular}

efficient (males and females) of $0.73(P<0.001)$ was obtained. However, the correlation coefficient was significantly higher for males than for females $(r=0.82, r=$ 0.55 respectively, with $P<0.05$ ).

The correlation between the anaesthetists' assessment of the patients' anxiety at the afternoon visit and the projected level of anxiety with the STAI and MAACL scores were extremely low (Tables II and III). There was no difference between staff and resident anaesthetists' abilities to predict anxiety. There were also no differences when patients were subdivided according to their sex, age (greater than or less than 40 ), or the type of surgical procedure being performed. There were similar low correlations between the anaesthetists' assessments and the trait scores $(r=0.08)$, depression scores $(r=0.05)$ and the hostility scores $(r=0.22)$, indicating that anaesthetists were not confusing these with state anxiety. In contrast, differences $(P<0.01)$ were found between the correlations obtained for anaesthetists who asked their patients how anxious they felt $(r=0.66)$ and those who relied solely on physical and/or visual criteria $(r=0.01)$.

\section{Discussion}

This study substantiated the recent finding that patient anxiety on the afternoon before surgery correlates with that occurring immediately preoperatively. ${ }^{6}$ This same study claimed that no other factor (age, sex, previous surgery, sleeping pill use) could predict preoperative anxiety. However, we did show that females have more anxiety than males, and that lack of a previous surgical experience is also associated with increased anxiety. We studied 45 males and 39 females whereas Lichtor et al.

TABLE III Correlations between anaesthetist prediclive ability and test score immediately preoperatively

\section{Correlation coefficients}

Anaesthetist predictive scores

\begin{tabular}{llll}
\cline { 2 - 4 } STAI & Combined & Staff & Residents \\
\hline Score & 0.23 & 0.23 & 0.24 \\
\hline
\end{tabular}

only studied 12 men and 40 women. ${ }^{6}$ This sampling difference may explain their inability to show a difference based on sex. The influence of previous surgery on anxiety has also previously been shown by others. ${ }^{1,8}$

Several studies have shown that the majority of patients undergoing elective surgery have increased anxiety. ${ }^{1-3}$ This increased anxiety has been shown to correlate with increased postoperative pain, ${ }^{4,9}$ as well as with increased postoperative analgesic requirements and prolonged hospital stay. ${ }^{5}$ This morbidity could be minimized by decreasing anxiety through the use of anxiolytic premedication. Appropriate use of this medication can only be made if one can determine a patient's level of anxiety during the preoperative visit.

Our study, however, indicates that anaesthetists as a group are poor at assessing and predicting preoperative anxiety when compared with objective questionnaires completed by the patient. This should not be surprising as previous studies involving surgeons, ${ }^{3}$ psychologists ${ }^{10}$ and nurse-observers " have yielded similar results, i.e., objective tests outperform clinical judgement. A recent study ${ }^{12}$ did find correlations of 0.46 between the MAACL and HAD (hospital anxiety and depression scale) anxiety scores and anaesthetists' determination of patient anxiety. However, in that study the anaesthetists simply graded the patients' anxiety as absent, slight, moderate or marked. In contrast, we used the visual analogue scale (a continuous measure) which may be more sensitive than an interval scale and has been shown to correlate well with other objective tests of anxiety. ${ }^{7}$ These studies are in contrast to an earlier study which showed an 82 per cent agreement between patients' subjective responses and anaesthetists' objective findings. 'This assessment, however, consisted solely of either being "anxious" or "non-anxious." We feel, as do others, ${ }^{12-14}$ that a more objective measurement should be used. The Spielberger inventory and MAACL have been extensively validated as well as having been used in other perioperative anxiety studies. ${ }^{2,4,12,15-18}$ The fact that both questionnaire anxiety scores correlated with each other indicates an adequate level of convergent validity. Our correlation between the MAACL and the STAI is consistent with the findings of others. ${ }^{12,18}$

The ability of anaesthetists to assess preoperative anxiety accurately may be influenced by a number of factors. Since anaesthetists were not especially trained concerning the nature of anxiety, conceptualization of this emotion may have varied greatly and may not have corresponded to generally accepted definitions as reflected in the STAI and MAACL. A brief training session regarding the signs and symptoms of anxiety may increase predictive accuracy. Anaesthetists would also benefit from knowledge of common errors associated with such ratings (anxiety or other patient characteristics). ${ }^{10}$ Fre- 
quent sources of error include a tendency to be unduly biased by a single patient trait (positive or negative) which then colours the overall judgement of the person; a widespread tendency of raters to use middle categories and thereby avoid extreme judgments; and the common tendency of raters to be reluctant to assign what they perceive to be unfavourable ratings. An awareness of these sources of potential error could increase an anaesthetist's ability to detect anxiety accurately.

We conclude from the present data that anaesthestists are not accurate in asssessing patient anxiety but that predictive accuracy can be significantly improved when anaesthetists specifically ask patients whether or not they are anxious. This more forthright practice would allow more appropriate premedication and further decrease preoperative anxiety and its resulting sequelae. We have also confirmed that anxiety occurring the night before surgery remains constant into the immediate preoperative period.

\section{References}

I Norris W, Baird WLM. Preoperative anxiety - a study of the incidence and aetiology. Br J Anaesth 1967; 39: 503-9.

2 Johnson $M$. Anxiety in surgical patients. Psychol Med 1980; 10: 145-52.

3 Ramsay MAE. A survey of preoperative fear. Anaesthesia 1972; 27: 396-402.

4 Jamison RN, Parris WLV, Maxson WS. Psychological factors influencing recovery from outpatient surgery. Behav Res Ther 1987; 25: 31-3.

5 Wallace $L M$. Psychological preparation as a method of reducing the stress of surgery. J Hum Stress 1984; 10: 62-77.

6 Lichtor JL, Johanson CE, Mhoon D, Faure EAM, Hassan $S Z$, Roizen $M F$. Preoperative anxiety - does anxiety level the afternoon before the surgery predict anxiety level just before surgery? Anesthesiology 1987; 67: 595-9.

7 Arellano $R$, Cruise $C$, Chung $F$. Timing of the anesthetist's preoperative outpatient interview. Anesth Analg 1989; 68: 645-8.

8 Egbert LD, Battil GE, Turndorf H, Beecher HK. The value of the preoperative visit by an anesthetist. JAMA 1963; 185: 553-5.

9 Scot LE, Clum GA, Peoples JB. Preoperative predictors of postoperative pain. Pain 1983; 15: 283-93.

10 Wiggins JS. Personality and Prediction. Principles of Personality Assessment. Reading, Massachusetts: AddisonWesley, 1973.

11 Forrest WH, Brown CR, Brown BW. Subjective responses to six common preoperative medications. Anesthesiology 1977; 47: 241-7.

12 Hicks JA, Jenkins JG. The measurement of preoperative anxiety. J Roy Soc Med 1988; 81: 517-9.
13 Madej TH, Pasuuke RT. Anaesthetic premedication: aims, assessment and methods. Can J Anaesth 1987; 34: 259-73.

14 Whire $P F$. Pharmacologic and clinical aspects of preoperative medication. Anesth Analg 1986; 65: 963-74.

15 Leigh JM, Walker J, Janaganathan P. Effect of preoperative anaesthetic visit on anxiety. Br Med J 1977; 2 987-9.

16 Stallworth JM, Martino-Saltzman D. Comparison of benzodiazepine premedications triazolam and diazepam amnesia, anxiolysis and sedation. Anesth Analg 1987; 66: S165.

17 Wassenaar W, Lancee WJ, Galloon S, Gale GD. The measurement of anxiety in the pre-surgical patient. $\mathrm{Br} J$ Anaesth 1977; 49: 605-8.

18 Spielberger $C D$. State-Trait Anxiety Inventory: A Comprehensive Bibliography. Palo Alto, Consulting Psychologists Press Inc., 1984. 